\title{
Nanoscale
}

Check for updates

Cite this: Nanoscale, 2017, 9, 12727

\section{Nanoparticle discrimination based on wavelength and lifetime-multiplexed cathodoluminescence microscopy}

\author{
Mathijs W. H. Garming, ${ }^{a}$ I. Gerward C. Weppelman, ${ }^{\text {a Pascal de Boer, }}{ }^{\text {b }}$ \\ Felipe Perona Martínez, ${ }^{c}$ Romana Schirhagl, (D) ${ }^{c}$ Jacob P. Hoogenboom (D) *a and \\ Robert J. Moerland (D)*a
}

\begin{abstract}
Nanomaterials can be identified in high-resolution electron microscopy images using spectrally-selective cathodoluminescence. Capabilities for multiplex detection can however be limited, e.g., due to spectral overlap or availability of filters. Also, the available photon flux may be limited due to degradation under electron irradiation. Here, we demonstrate single-pass cathodoluminescence-lifetime based discrimination of different nanoparticles, using a pulsed electron beam. We also show that cathodoluminescence lifetime is a robust parameter even when the nanoparticle cathodoluminescence intensity decays over an order of magnitude. We create lifetime maps, where the lifetime of the cathodoluminescence emission is correlated with the emission intensity and secondary-electron images. The consistency of lifetime-based discrimination is verified by also correlating the emission wavelength and the lifetime of nanoparticles. Our results show how cathodoluminescence lifetime provides an additional channel of information in electron microscopy.
\end{abstract}

Received 7th February 2017 Accepted 10th August 2017 DOI: $10.1039 / \mathrm{c} 7 \mathrm{nr} 00927 \mathrm{e}$ rsc.li/nanoscale ticularly from organic molecules, under electron irradiation. ${ }^{18}$ Cathodoluminescent nanoparticles (CNPs), for example phosphors composed of metal oxides ${ }^{13,19-21}$ and fluorescent nanodiamonds (FNDs), ${ }^{22}$ are an attractive alternative for labeling in biological sciences with size and thus resolution that can go down to a few nanometers. ${ }^{23-25}$ Multicolor experiments can be realized by employing various types of emitters, such as rare earth ions ${ }^{13}$ and FNDs with engineered defects to modify the emission spectrum. ${ }^{22}$ However, as in fluorescence microscopy, the analytical power of spectral discrimination is inherently limited due to overlap of emission bands and availability of filters.

In fluorescence microscopy, lifetime imaging ${ }^{26,27}$ provides additional or otherwise unobtainable data, on, for example, the local (optical) environment ${ }^{28-30}$ or state of fluophores. ${ }^{31}$ Moreover, lifetime imaging can be used to differentiate between various emitters. ${ }^{26}$ With strong CL signals, the fluorescence lifetime can be obtained by fitting exponential curves to decay curves after excitation with continuous beams. ${ }^{32,33}$ For low signals, single photon counting can still accurately resolve lifetimes by determining, e.g., antibunching curves ${ }^{34}$ or photon arrival time histograms. ${ }^{35}$ The latter option can be used to minimize electron-induced damage to the sample by exciting the sample with short electron pulses, while also maintaining the native resolution of the Scanning EM $(\mathrm{SEM}) \cdot{ }^{36,37}$

\footnotetext{
${ }^{a}$ Delft University of Technology, Lorentzweg 1, NL-2628CJ Delft, The Netherlands. E-mail:j.p.hoogenboom@tudelft.nl, r.j.moerland@tudelft.nl

${ }^{b}$ University Medical Center Groningen, Department of Cell Biology, Antonius Deusinglaan 1, NL-9713AW Groningen, The Netherlands ${ }^{c}$ University Medical Center Groningen, Department of Biomedical Engineering, Antonius Deusinglaan 1, NL-9713AW Groningen, The Netherlands
} 
Here, we demonstrate the ability to discriminate between various CNPs in the time domain. We show that we can measure the emission intensity of CNPs while exciting them with a pulsed electron beam, which is generated by a commercial beam blanker. ${ }^{37}$ For each pixel in the intensity map we measure the intrinsic lifetime associated with the type of emitter at that particular location. To confirm our discrimination based on the time-domain data, we measure spectrallyselective CL intensity maps for comparison.

\section{Results \& discussion}

We have modified a standard electron microscope to include a high-NA optical objective, which is part of an inverted optical microscope, ${ }^{14,38,39}$ and have added a pulsed electron-beam mode suitable for time-resolved imaging (see Fig. 1). With this setup, we obtained electron pulses with lengths down to 80-90 ps. We employ a Time-Correlated Single-Photon Counting (TCSPC) scheme (PicoQuant PicoHarp 300), which is often used in fluorescence microscopy to determine the fluorescence lifetime of fluophores. In that case, the TCSPC clock signal is typically issued by a pulsed laser. Here we drive the blanker with a pulse generator and use the same signal to synchronize with the TCSPC hardware.

Samples with dispersed CNPs are obtained by spincoating from solution. In order to establish the distribution of lifetimes of each type of nanoparticle, we first prepare two samples, where each sample contains only one type of particle. Here, we use cerium-doped $\mathrm{Lu}_{3} \mathrm{Al}_{5} \mathrm{O}_{12}$ (LuAG:Ce) particles (Boston Applied Technology, Inc.) that exhibit a transition from the $5 \mathrm{~d}$ level to the $4 \mathrm{f}$ ground state ${ }^{40}$ of $\mathrm{Ce}^{3+}$, and FNDs (Adamas) that exhibit a transition from the ${ }^{2} \mathrm{~A}$ state to the ${ }^{2} \mathrm{E}$ state, ${ }^{41}$ associated with the display of CL by primarily the neutral $\mathrm{NV}^{0}$ center. ${ }^{42}$ Typical photon arrival time histograms are shown in Fig. 2a. We approximate the measured arrival times with a single exponential, which yields a typical decay time of the CNP. We collect the decay time of 28 FNDs and 46 LuAG:Ce CNPs and determine the distribution of lifetimes

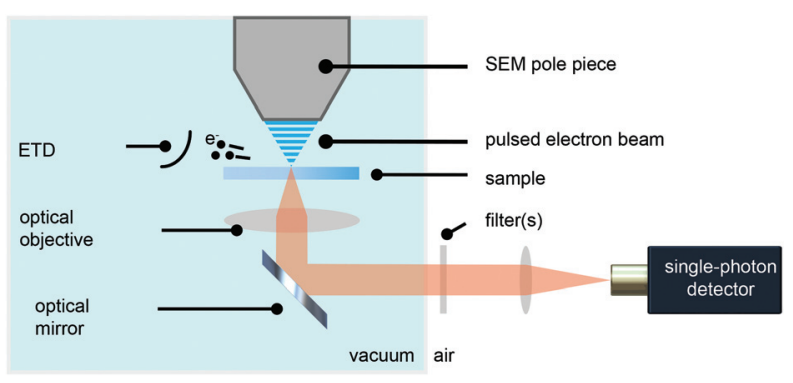

Fig. 1 Schematic depiction of an integrated optical and electron microscope. A pulsed electron beam strikes the sample with a fixed frequency. An Everhart-Thornley detector (ETD) collects secondary electrons used for imaging with the SEM. Cathodoluminescence, induced by the energetic electrons, is collected with a 0.95 NA optical objective and imaged onto a single-photon counting detector.
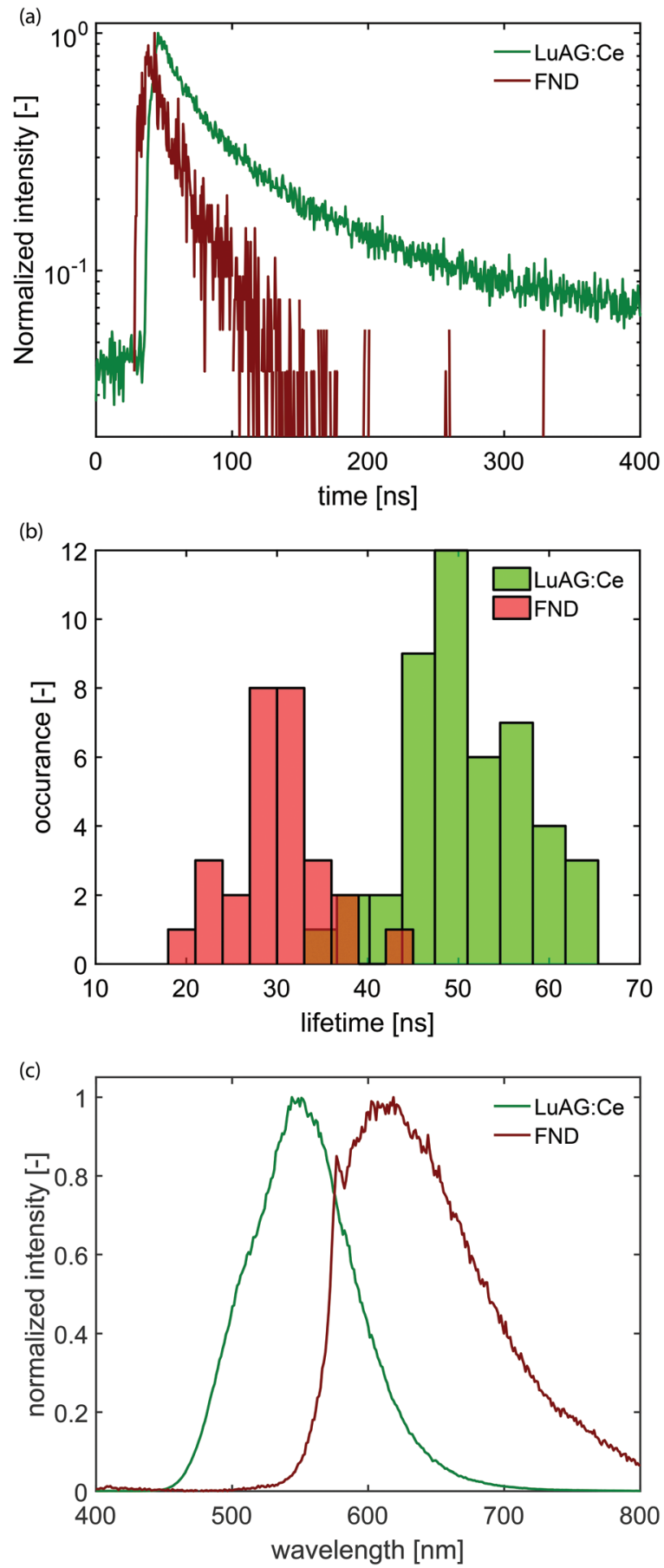

Fig. 2 Emission properties of the two types of particles used here. (a) Typical photon arrival time histograms for $\mathrm{Lu}_{3} \mathrm{Al}_{5} \mathrm{O}_{12}: \mathrm{Ce}^{3+}$ (LuAG:Ce) particles (green) and fluorescent nanodiamonds (FNDs, red). (b) Histogram of lifetimes of various nanoparticles, as obtained by timecorrelated single-photon counting. The average lifetime of FNDs is 30 ns with a width of $10 \mathrm{~ns}(2 \sigma)$. The average lifetime of the LuAG:Ce par-

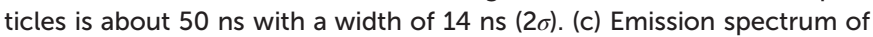
LuAG:Ce (green) and FNDs (red).

(Fig. 2b). The difference in lifetime distribution between the two particles is clearly visible; LuAG:Ce nanoparticles have a typical lifetime of about $50 \mathrm{~ns}$, with a width of $14 \mathrm{~ns}(2 \sigma)$. The 
FNDs on the other hand have a substantially shorter lifetime of typically $30 \mathrm{~ns}$, with a width of $10 \mathrm{~ns}(2 \sigma)$. Finally, in Fig. 2c, the measured emission spectra of both types of CNPs are shown.

\section{Stability of lifetime under e-beam irradiation}

Next, we investigated the stability of the lifetime measurements over the irradiation time. In contrast to intensity, which changes over time during the irradiation with charged particles, lifetime is a relatively stable quantity. This is highlighted in Fig. 3a-d. Fig. 3a displays the measured lifetime of three FNDs over a time span of 10 minutes. The photon arrival histograms were determined every 5 seconds. The number of photons in every histogram is displayed in Fig. $3 \mathrm{~b}$ over the same time span. Similar measurements for the LuAG:Ce particles are shown in Fig. $3 \mathrm{c}$ and d. Despite the fact that the CL intensity varies (likely due to bleaching and drift of the electron beam), the measured lifetime remains extremely robust. As a particularly extreme example, the lifetime of the FND displayed with a blue curve in Fig. 3a varies between a maximum of $27 \mathrm{~ns}$ and a minimum of $20 \mathrm{~ns}$ and has a mean of $25.0 \mathrm{~ns} \pm$ $12 \%(3 \sigma)$. This happens while the intensity drops by a factor of 29. Whereas bleaching of FNDs has been reported in literature $^{43}$ the exact mechanism is still subject of research. Potentially the creation or annihilation of surface charge traps,${ }^{44}$ dynamics of impurities or charge carriers in the vicinity of the vacancy centers, ${ }^{45-48}$ or quenching of nitrogenvacancy centers near the surface by a thin carbon contamination layer grown by e-beam-induced deposition play a role. This is subject to further investigation.

\section{Cathodoluminescence lifetime maps}

Subsequently, a sample is prepared that contains both types of CNPs, with approximately a $1: 1$ ratio of the number of particles of each type. A secondary-electron (SE) image of a $6 \times 6 \mu \mathrm{m}$ region on the sample is displayed in Fig. $4 \mathrm{a}$. We scan the same area again, but now with a pulsed electron beam at a step size of $100 \mathrm{~nm}$ and a dwell time of 0.5 seconds. For every pixel, the photon arrival histogram is determined, which is then fitted with a single exponential decay. Thus, we simul-
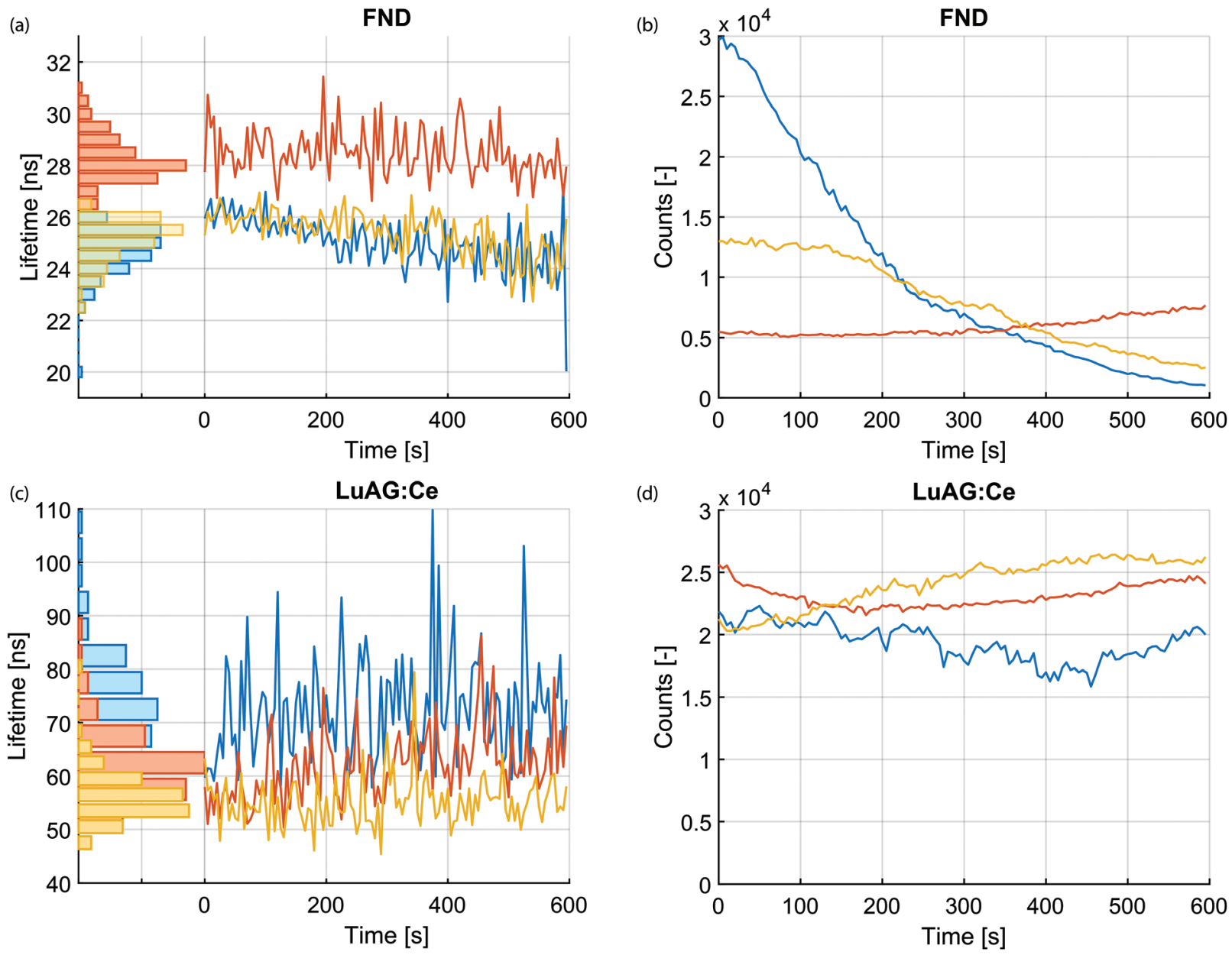

Fig. 3 Stability of lifetime and intensity versus irradiation with the electron beam, for three particles of each type (LuAG:Ce and FND). Lifetime versus e-beam irradiation duration, determined every 5 seconds, of three FNDs and LuAG:Ce particles is shown in panel (a) and (c), respectively. Panels (b) and (d) show the corresponding CL intensity during the measurement. Despite (severe) differences in intensity, the lifetime of the nanoparticles is a robust parameter during the measurement. 

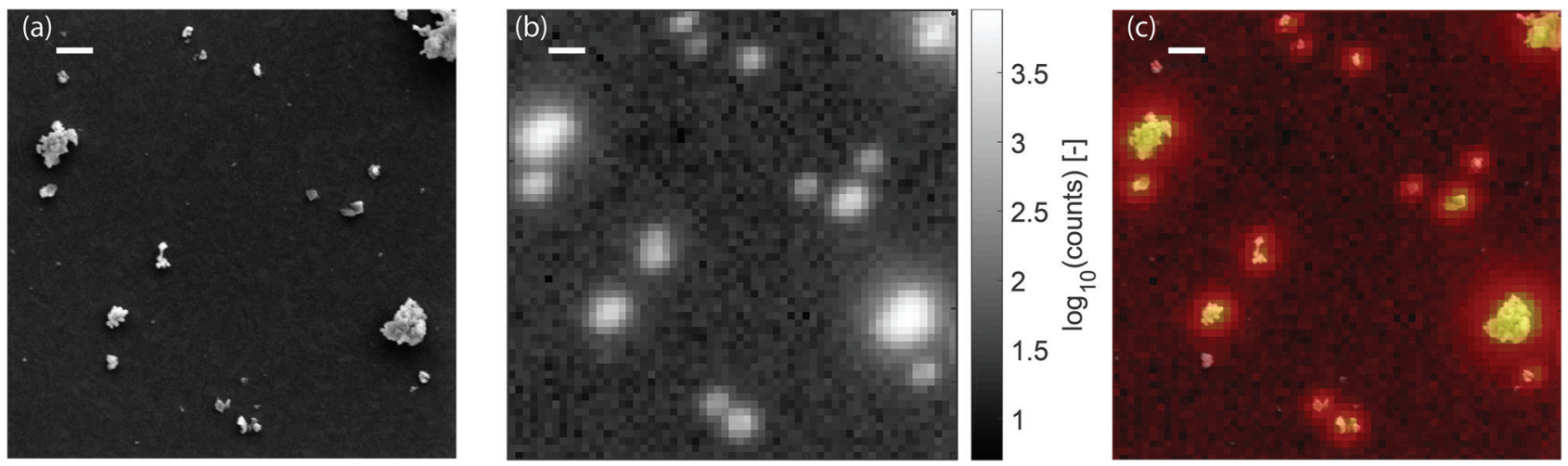

Fig. 4 (a) Secondary electron (SE) image a typical area of a sample containing a mixture of LuAG:Ce and FND nanoparticles. The nanoparticles are deposited onto a glass cover slide with a thin indium-tin-oxide coating which provides the optical transparency and electric conductance required. In (b), the cathodoluminescence $(\mathrm{CL})$ is displayed on a logarithmic scale. In (c), a false-color image where the SE image and the (logarithmic) $\mathrm{CL}$ intensity image are overlaid is shown, showing the clear correlation between the two.

taneously measure the intensity and lifetime of the emitters on the sample. The intensity of both type of particles can differ greatly, which on a linear scale could render the less intense particles invisible. Therefore, we show the CL intensity map in Fig. 4b on a logarithmic scale. Note that this leads to an apparently larger size of the particle. The correlation between the CL intensity and the SE image can be seen in Fig. $4 \mathrm{c}$, where the intensity is shown in a false color scale for identification purposes only.

The lifetime map that we obtain from the fitting procedure is shown in Fig. 5a. Here, the lifetime map is thresholded with the intensity, such that areas without CNPs do not show as noise in the lifetime maps. Clearly, there are two dominant lifetimes in Fig. 5a, corresponding to the distribution of lifetimes as measured before and displayed in Fig. 2b. Particles with a lifetime of around 20-30 ns correspond to FNDs, and particles with a lifetime of about $50-70$ ns correspond to LuAG:Ce particles. For comparison, the scans were repeated, inserting optical filters to discriminate between the two particle types: a short pass filter with an edge wavelength of $550 \mathrm{~nm}$ in order to only see LuAG:Ce particles, and a long pass filter in order to see the emission by the FNDs. Due to the broad spectrum and high CL intensity, the LuAG:Ce particles are suppressed in intensity but visible as well. The results for the short pass filter are shown in Fig. 5b, whereas the results for the long pass filter are shown in Fig. 5c. As an example, the low-lifetime FNDs highlighted by a white square in Fig. 5a clearly only show up in the longer-wavelength emission channel in Fig. 5c, corresponding to the bulk of the emission spectrum of FNDs. Thus, the spectral data confirm the validity of our CL lifetime-based discrimination.

For higher resolution CL lifetime imaging, the step size of the electron beam can be reduced. In Fig. 6 we show three different nanoparticles scanned with a step size of $50 \mathrm{~nm}$. As the current with pulsed electron beam operation is reduced, image acquisition times are longer compared to continuous
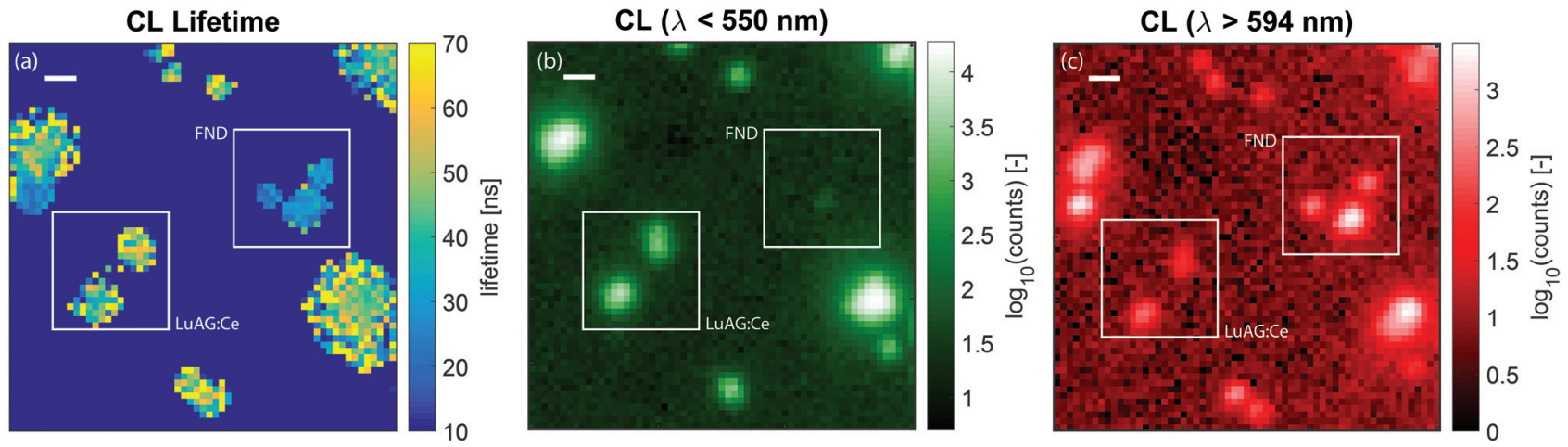

Fig. 5 Lifetime and spectral maps of LUAG and FND nanoparticles. In (a), the measured lifetime as a function of position is shown, with typical times around 40-60 ns for LuAG:Ce particles and around 20-30 ns for FNDs. The squares highlight examples of the type of particle as determined by the lifetime and the histograms of Fig. 2b. Here, no wavelength selection was applied. In (b), the CL intensity for light with wavelengths less than $550 \mathrm{~nm}$ is shown, corresponding to the emission of LuAG:Ce particles. In (c), the CL intensity for light with wavelength longer than $594 \mathrm{~nm}$ is shown, corresponding to a tail in the emission by LuAG:Ce and the bulk of the FND emission. The FNDs are absent in panel (b) and clearly show up in panel (c). The broad spectrum of LuAG:Ce means that this type of particle shows up in both measurements. However, for wavelengths longer than $597 \mathrm{~nm}$ the intensity is greatly reduced. In panel (a)-(c), the scale bar is $500 \mathrm{~nm}$. 


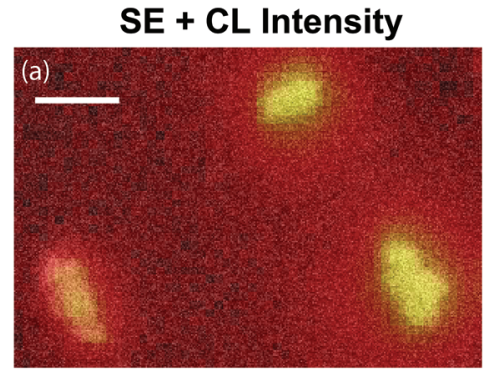

$\operatorname{CL}(\lambda<550 \mathrm{~nm})$

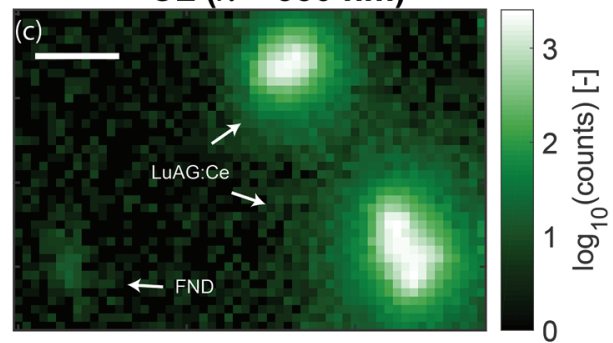

CL Lifetime

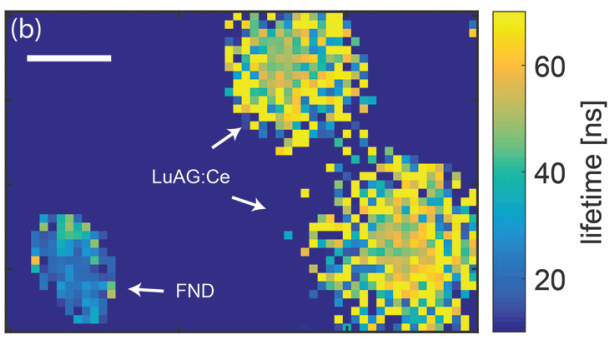

$\operatorname{CL}(\lambda>594 \mathrm{~nm})$

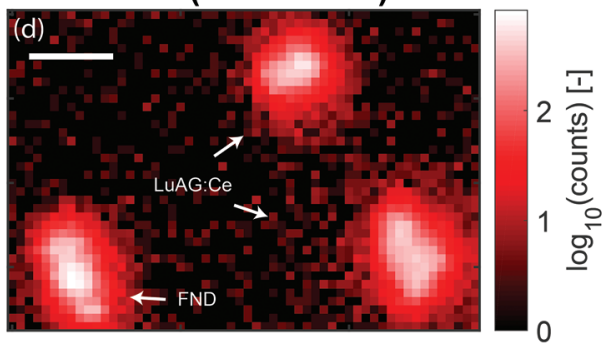

Fig. 6 High-resolution lifetime and spectral maps of LuAG:Ce and FND nanoparticles. (a) False-color image of SE overlaid with the (logarithmic) CL intensity. (b) Measured lifetime as a function of position. The arrows indicate the two types of particles as determined by the lifetime and the histograms of Fig. 2b. No wavelength selection was applied. (c) CL intensity for light with wavelengths less than $550 \mathrm{~nm}$ is shown (LuAG:Ce particles). (d) CL intensity for light with wavelength longer than $594 \mathrm{~nm}$ is shown (bulk of the FND emission). The FNDs are nearly absent in panel (c) and clearly show up in panel (d). In panel (a)-(d), the scale bar is $500 \mathrm{~nm}$.

operation and thus choice of step size is a balance between resolution required for particle identification and field of view size. The results shown in Fig. 6 confirm the above conclusion that we can discriminate the particles based on lifetime, where here we are limited by the particle size.

\section{CL lifetime in stained biological sections}

Application of CL lifetime imaging for nanostructural characterization may range from geosciences and materials sciences, phosphor particle detector development, to visualization of labels in biological sciences. As an application example, we here demonstrate that CL lifetime imaging can be conducted on biological sections after fixation, staining, and sectioning protocols. Fig. 7a shows a back-scattered electron (BSE) image of a $300 \mathrm{~nm}$ section of macrophages incubated with the FNDs. The image shows good ultrastructural preservation and fine intracellular details are well resolved. The FNDs are however not visible in the BSE images. In Fig. 7b and $\mathrm{c}$ we show a zoom of an area where CL was detected, presumably from uptaken FNDs. The CL lifetime map in Fig. 7e indeed unequivocally

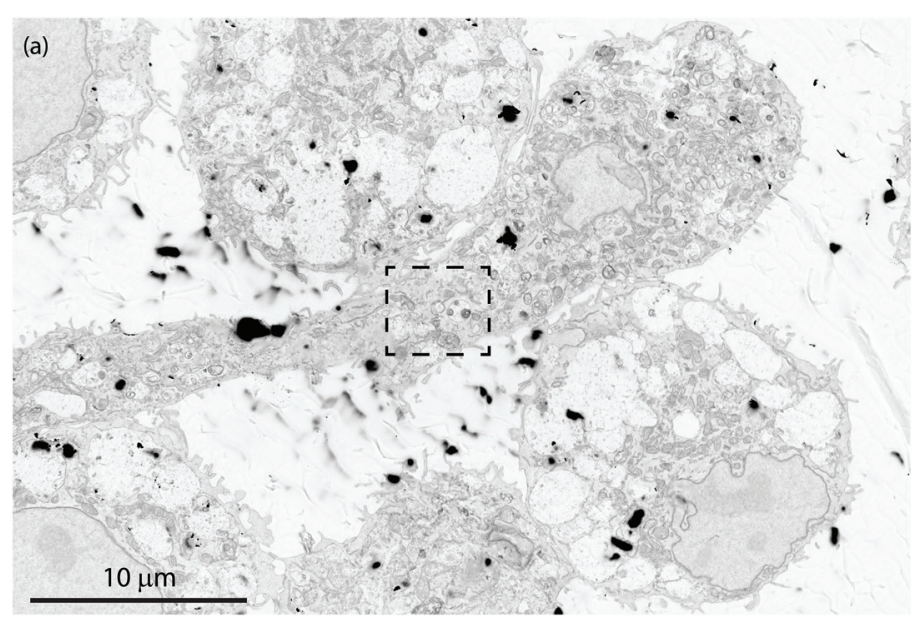

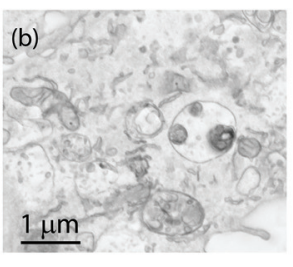

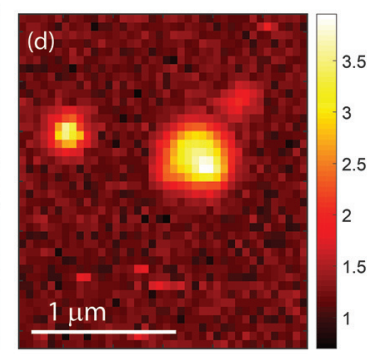

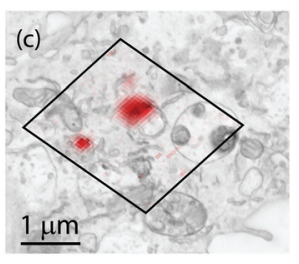

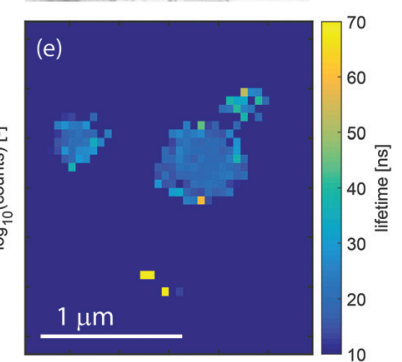

Fig. $7 \mathrm{CL}$ lifetime measurements of FNDs in stained sections after uptake in macrophages. (a) Backscattered electron image of part a section showing macrophage ultrastructure (b) zoom-in of the dashed area in (a), which is a region where CL was detected.(c) Logarithmic CL intensity map from area (b) overlaid on the BSE image. (d) Logarithmic CL intensity, and (e) simultaneously acquired CL lifetime map for the area in (b), clearly showing $C L$ lifetime values typical for FNDs. 
identifies the FNDs as being the source of the CL emission logarithmically plotted in Fig. 7d ( $c f$. Fig. 2b). The weaker CL signal for one of the three FNDs may be due to an FND buried deeper within the $300 \mathrm{~nm}$ section. Indeed, while the other two particles stood out in the SE image as well (data not shown), as expected for surface-exposed FNDs, ${ }^{25}$ no SE signal above background tissue signal was recorded at this location. The BSE-CL overlay allows for high-resolution localization of the CNPs in the macrophage ultrastructural environment and lifetime data is useful to identify these as FNDs.

\section{Conclusions}

We have shown that lifetime-based imaging and particle discrimination is a stable modality in electron microscopy, useful for analytical and correlative imaging purposes. Based on lifetime, we demonstrated discrimination of particle type in a mixture of two CNPs dispersed over a surface, and CL lifetime imaging of CNPs in tissue sections. The lifetime of the CNPs measurements show very good robustness over time, with only mild variations even when the intensity itself diminishes significantly.

For applications, we believe that correlative SE and CL lifetime imaging is suitable for high-resolution characterization and imaging throughout the nanosciences. Particular areas of interest may include materials sciences and geosciences in general, and characterization of the homogeneity of phosphors for use in particle detectors or of nanoparticle assemblies in particular. In addition, the pulsed electron excitation paired with the possibility to obtain accurate lifetime estimates from histograms with low photon counts ${ }^{35}$ may be particularly beneficial for systems suffering from electron-beam degradation, such as labels in biological tissue sections. Moreover, instead of or combined with multi-color CL imaging, the lifetime of the nanoparticles may be used to increase the number of labels discernible in EM; e.g., green-fluorescing nanodiamonds and LuAG:Ce have very similar optical emission spectra but have different lifetimes, ${ }^{49}$ which is sufficient to distinguish the particles. Time-resolved CL imaging can also be beneficial in combination with other characterization techniques such as energy-dispersive X-ray spectroscopy ${ }^{50}$ e.g., to distinguish materials with similar elemental composition such as different (carbon-based) FNDs and native biological tissue or polymeric materials. Finally, CL lifetime microscopy may be used to recover more information about the local environment of the nanoparticle, such as structures which modify the optical local density of states, ${ }^{37,51}$ or the actual (surface) structure or doping concentration of the nanoparticle itself. ${ }^{52-54}$

\section{Methods}

\section{Particle samples}

Sample preparation. The sample containing both types of CNPs was prepared by a combination of spincoating and drop- casting particles in solution on an indium-tin oxide (ITO) covered glass slide. The Ce doped LuAG particles were dispersed in ethanol at a concentration of $2 \mathrm{mg} \mathrm{ml}^{-1}$ and the solution was spincoated on the ITO at $1000 \mathrm{rpm}$ for 2 minutes. To ensure sufficiently dense coverage, the spincoating was done five times consecutively. The DI water based solution in which the FNDs are supplied was diluted by a factor 20 for a concentration of approximately $0.05 \mathrm{mg} \mathrm{ml}^{-1}$. A $10 \mu \mathrm{l}$ droplet was deposited on the LuAG coated ITO slide and left to dry on a hot plate at about $50{ }^{\circ} \mathrm{C}$. Sample containing only a single particle variety were prepared in the same manner, omitting deposition of the other CNP type.

SE and CL measurements. We used a beam energy of $4 \mathrm{kV}$ in conjugated blanking mode $\mathrm{e}^{37}$ to excite the CNPs and measure SEs. The current in the continuous electron beam was $19 \mathrm{pA}$, and while in this mode we retrieved the SE image of the sample surface. Then, we switched on the pulse generator to drive the beam blanker at a repetition rate of $500 \mathrm{kHz}$. This results in two pulses per cycle; one at the rising edge of the pulse and one at the falling edge. Therefore, a total measurement time of $1 \mu$ s after each electron pulse is available to build the photon arrival histogram. With longest decay times of about $90 \mathrm{~ns}$, this is sufficient to faithfully retrieve the arrival histograms. An avalanche photodiode (PicoQuant) was used to collect the photons emitted by the sample.

\section{Macrophage samples}

Sample preparation. For the uptake experiments, ground HPHT diamonds (Adamas Nano; on average $120 \mathrm{~nm}$ hydrodynamic radius and $900 \mathrm{NV}$ centers per particle as stated by supplier) were used. The particles were coated with a protein polymer consisting of a C4 domain and a K12 domain. C4 is a tetramer of a 98 amino acid long, hydrophilic random coil polypeptide, which is rich in glycines, prolines and other hydrophilic amino acids. ${ }^{55}$ K12 consists of 12 lysines which are positively charged. The coating not only improves colloidal stability but the positive charge also might favor formation of NV0. The C4K12 protein polymers were obtained as described elsewhere. ${ }^{55}$ In short, secreted expression by genetically engineered Pichia pastoris strains was used. The protein was synthesized with a so-called methanol fed-batch fermentation. Then the protein-containing supernatant was separated from the $P$. pastoris cells by centrifugation (30 $\mathrm{min}$ at $16000 \mathrm{~g}$ ) and subsequent microfiltration. The protein was purified by selective precipitation with ammonium sulfate (at $45 \%$ saturation). After a dialysis against $50 \mathrm{mM}$ formic acid the protein was lyophilized and the protein powder was stored at room temperature. Before coating the polymer was diluted in MilliQ water to a concentration of $7.36 \mathrm{mg} \mathrm{ml} \mathrm{m}^{-1}$. The protein was passed through a $0.22 \mu \mathrm{m}$ filter to remove aggregates.

The diamond particles were coated with the C4K12 protein polymers by mixing the stock solution of nanodiamonds $\left(100 \mu \mathrm{g} \mathrm{mL} \mathrm{m}^{-1}\right.$ in water), with the protein solution followed by incubating for 30 minutes. The final particles contain roughly 4 C4K12 molecules per $100 \mathrm{~nm}^{2}$ of nanodiamond surface. All solutions were prepared under sterile conditions. 
J774A.1 macrophages (LGC Standards, Germany) were cultured in Dulbecco's Modified Eagle Medium (DMEM), supplemented with $10 \% \mathrm{FBS}, 1 \%$ penicillin/streptomycin. Cells were incubated with $1 \mu \mathrm{g} \mathrm{ml}^{-1}$ coated FND in cell culture medium for 5 hours at $37{ }^{\circ} \mathrm{C}$ and $5 \% \mathrm{CO}_{2}$. After removal of culture medium with FNDs, cells were fixed with $4 \%$ paraformaldehyde/ $0.1 \%$ glutaraldehyde in $0.1 \mathrm{M}$ cacodylate buffer, $\mathrm{pH} 7.4$ for 30 minutes at room temperature. After washing with $0.1 \mathrm{M}$ cacodylate buffer, cells were incubated with $1 \%$ osmiumtetroxide/1.5\% potassiumferrocyanide in $0.1 \mathrm{M}$ cacodylate buffer for 30 minutes at $4{ }^{\circ} \mathrm{C}$, followed by washing with water. Next, the cells were dehydrated through an increasing graded ethanol series and left overnight in 1:1 ethanol and Epon (Serva) mixture at room temperature, which was replaced by pure Epon (4 times) and finally polymerised overnight at $58{ }^{\circ} \mathrm{C}$. The cover glass of the imaging dish was removed using hydrogen fluoride. Areas containing cells were selected using a stereo microscope and sawn from the Epon block. Subsequently, $300 \mathrm{~nm}$ sections were cut with an ultramicrotome (Leica EM UC7) using a glass knife and put on an ITO-coated cover glass.

BSE, SE and CL lifetime measurements. Lifetime and CL measurements on FNDs incorporated in macrophages have been performed with an FEI Quanta FEG 200 SEM by scanning the sample with a pulsed electron beam with a step size of $50 \mathrm{~nm}$ and a dwell time of $1 \mathrm{~s}$. The acceleration voltage and beam current without using the beam blanker were $3 \mathrm{kV}$ and $13 \mathrm{pA}$, respectively. At every electron-beam position, the SE signal, CL intensity, and photon arrival time histograms were simultaneously recorded and lifetime values were obtained from single exponential fits to the histograms.

As BSE detection provides better contrast on biological tissue than SE detection, and our time-resolved setup is not equipped with a BSE detector, the biological structure of the cell was imaged with an FEI Verios 460 SEM. By referring to the SE images obtained during the time-resolved measurement, the region of interest is retrieved, and SE and BSE images are simultaneously obtained with a $3 \mathrm{kV}, 0.8 \mathrm{nA}$ continuous electron beam.

BSE and CL image overlay. Both type of measurements described above, i.e., the retrieval of the BSE and CL lifetimes, also result in an SE image. Therefore, we used the SE images as ground truth data and registered the SE channels from both independent measurements onto each other. Here, we assumed an affine transformation. After the registration, the same transformation is used to map the BSE and CL images onto each other, resulting into Fig. 7c. The registration was performed with Image $^{56}$ together with the TurboReg plugin. $^{57}$

\section{Conflicts of interest}

Our integrated microscope and cathodoluminescence detection set-up served as a prototype for a product by Delmic BV. $\mathrm{JPH}$ is co-founder and shareholder in Delmic.

\section{Acknowledgements}

This research was supported by the Netherlands Organization for Fundamental Research (NWO) and the Stichting voor Fundamenteel Onderzoek der Materie (FOM, Foundation for Fundamental Research on Matter) grant number 10PR2826. RS acknowledges support from NWO-FOM via grant number FOM-G-36, PdB from Stichting Technische Wetenschappen (STW) - Perspectief voor de Topsectoren - Microscopy Valley grant number 12718, project leader Ben Giepmans. FPM is supported by A CONICYT scholarship from the Chilean government. Part of the work has been performed in the UMCG Microscopy and Imaging Center (UMIC), sponsored by ZonMW grant 91111.006 and NWO 175-010-2009-023.

\section{References}

1 E. F. Bond, D. Beresford and G. H. Haggis, J. Microsc., 1974, 100, 271-282.

2 B. G. Yacobi and D. B. Holt, J. Appl. Phys., 1986, 59, R1R24.

3 D. Poelman, D. Wauters, J. Versluys and R. L. V. Meirhaeghe, J. Appl. Phys., 2001, 90, 248-251.

4 K. Milliken, S.-J. Choh, P. Papazis and J. Schieber, Sediment. Geol., 2007, 198, 221-232.

5 B. E. Sørensen and R. B. Larsen, Contrib. Mineral. Petrol., 2008, 157, 147.

6 M. Kostova, M. Batentschuk, F. Goetz-Neunhoeffer, S. Gruber, A. Winnacker, P. Greil and C. Zollfrank, Mater. Chem. Phys., 2010, 123, 166-171.

7 J. Bruckbauer, P. R. Edwards, T. Wang and R. W. Martin, Appl. Phys. Lett., 2011, 98, 141908.

8 M. F. Hamers and M. R. Drury, Meteorit. Planet. Sci., 2011, 46, 1814-1831.

9 P. V. Hough, W. R. McKinney, M. C. Ledbeter, R. E. Pollack and H. W. Moos, Proc. Natl. Acad. Sci. U. S. A., 1976, 73, 317-321.

10 J. Silver, R. Withnall, T. G. Ireland, G. R. Fern and S. Zhang, Nanotechnology, 2008, 19, 095302.

11 M. Kuttge, E. J. R. Vesseur, A. F. Koenderink, H. J. Lezec, H. A. Atwater, F. J. García de Abajo and A. Polman, Phys. Rev. B: Condens. Matter, 2009, 79, 113405.

12 B. Dierre, X. Yuan and T. Sekiguchi, Sci. Technol. Adv. Mater., 2010, 11, 043001.

13 H. Niioka, T. Furukawa, M. Ichimiya, M. Ashida, T. Araki and M. Hashimoto, Appl. Phys. Express, 2011, 4, 112402.

14 A. C. Narváez, I. G. C. Weppelman, R. J. Moerland, N. Liv, A. C. Zonnevylle, P. Kruit and J. P. Hoogenboom, Opt. Express, 2013, 21, 29968-29978.

15 C. I. Osorio, T. Coenen, B. J. M. Brenny, A. Polman and A. F. Koenderink, ACS Photonics, 2016, 3, 147-154.

16 P. de Boer, J. P. Hoogenboom and B. N. G. Giepmans, Nat. Methods, 2015, 12, 503-513. 
17 D. R. Glenn, H. Zhang, N. Kasthuri, R. Schalek, P. K. Lo, A. S. Trifonov, H. Park, J. W. Lichtman and R. L. Walsworth, Sci. Rep., 2012, 2, 865.

18 J.-i. Niitsuma, H. Oikawa, E. Kimura, T. Ushiki and T. Sekiguchi, J. Electron Microsc., 2005, 54, 325.

19 K. Kömpe, H. Borchert, J. Storz, A. Lobo, S. Adam, T. Möller and M. Haase, Angew. Chem., Int. Ed., 2003, 42, 5513-5516.

20 Y. Nawa, W. Inami, A. Chiba, A. Ono, A. Miyakawa, Y. Kawata, S. Lin and S. Terakawa, Opt. Express, 2012, 20, 5629-5635.

21 T. Furukawa, H. Niioka, M. Ichimiya, T. Nagata, M. Ashida, T. Araki and M. Hashimoto, Opt. Express, 2013, 21, 2565525663.

22 Y. Nawa, W. Inami, S. Lin, Y. Kawata, S. Terakawa, C.-Y. Fang and H.-C. Chang, ChemPhysChem, 2014, 15, 721-726.

23 I. E. G. Morrison, A. Samilian, P. Coppo, T. G. Ireland, G. R. Fern, J. Silver, R. Withnall and P. J. O'Toole, J. Chem. Biol., 2015, 8, 169-177.

24 S. Nagarajan, C. Pioche-Durieu, L. H. G. Tizei, C.-Y. Fang, J.-R. Bertrand, E. Le Cam, H.-C. Chang, F. Treussart and M. Kociak, Nanoscale, 2016, 8, 11588-11594.

25 S. R. Hemelaar, P. de Boer, M. Chipaux, W. Zuidema, T. Hamoh, F. P. Martinez, A. Nagl, J. P. Hoogenboom, B. N. G. Giepmans and R. Schirhagl, Sci. Rep., 2017, 7, 720.

26 J. R. Lakowicz and K. W. Berndt, Rev. Sci. Instrum., 1991, 62, 1727-1734.

27 X. F. Wang, A. Periasamy, B. Herman and D. M. Coleman, Crit. Rev. Anal. Chem., 1992, 23, 369-395.

28 K. H. Drexhage, J. Lumin., 1970, 1-2, 693-701.

29 R. A. L. Vallée, N. Tomczak, L. Kuipers, G. J. Vancso and N. F. van Hulst, Phys. Rev. Lett., 2003, 91, 038301.

30 R. J. Moerland and J. P. Hoogenboom, Optica, 2016, 3, 112117.

31 P. R. Selvin, Nat. Struct. Biol., 2000, 7, 730-734.

32 D. Poelman and P. F. Smet, Physica B, 2014, 439, 35-40.

33 D. den Engelsen, P. G. Harris, T. G. Ireland, G. R. Fern and J. Silver, Ultramicroscopy, 2015, 157, 27-34.

34 S. Meuret, L. H. G. Tizei, T. Auzelle, R. Songmuang, B. Daudin, B. Gayral and M. Kociak, ACS Photonics, 2016, 3, 1157-1163.

35 M. Köllner and J. Wolfrum, Chem. Phys. Lett., 1992, 200, 199-204.

36 D. S. Yang, O. F. Mohammed and A. H. Zewail, Proc. Natl. Acad. Sci. U. S. A., 2010, 107, 14993-14998.

37 R. J. Moerland, I. G. C. Weppelman, M. W. H. Garming, P. Kruit and J. P. Hoogenboom, Opt. Express, 2016, 24, 24760-24772.

38 A. C. Zonnevylle, R. F. Van Tol, N. Liv, A. C. Narváez, A. P. Effting, P. Kruit and J. P. Hoogenboom, J. Microsc., 2013, 252, 58-70.
39 A. C. Narváez, I. G. C. Weppelman, R. J. Moerland, J. P. Hoogenboom and P. Kruit, Appl. Phys. Lett., 2014, 104, 251121.

40 H.-L. Li, X.-J. Liu and L.-P. Huang, Opt. Mater., 2007, 29, 1138-1142.

41 S. Felton, A. M. Edmonds, M. E. Newton, P. M. Martineau, D. Fisher and D. J. Twitchen, Phys. Rev. B: Condens. Matter, 2008, 77, 081201.

42 A. Zaitsev, Optical Properties of Diamond, Springer-Verlag, Berlin, Heidelberg, 2001.

43 C. Bradac, T. Gaebel, N. Naidoo, M. J. Sellars, J. Twamley, L. J. Brown, A. S. Barnard, T. Plakhotnik, A. V. Zvyagin and J. R. Rabeau, Nat. Nanotechnol., 2010, 5, 345-349.

44 R. Schirhagl, K. Chang, M. Loretz and C. L. Degen, Annu. Rev. Phys. Chem., 2014, 65, 83-105.

45 K.-M. C. Fu, C. Santori, P. E. Barclay and R. G. Beausoleil, Appl. Phys. Lett., 2010, 96, 121907.

46 L. Rondin, G. Dantelle, A. Slablab, F. Grosshans, F. Treussart, P. Bergonzo, S. Perruchas, T. Gacoin, M. Chaigneau, H.-C. Chang, V. Jacques and J.-F. Roch, Phys. Rev. B: Condens. Matter, 2010, 82, 115449.

47 K. Beha, A. Batalov, N. B. Manson, R. Bratschitsch and A. Leitenstorfer, Phys. Rev. Lett., 2012, 109, 097404.

48 C. Zachreson, A. A. Martin, I. Aharonovich and M. Toth, ACS Appl. Mater. Interfaces, 2014, 6, 10367-10372.

49 G. Laporte and D. Psaltis, Biomed. Opt. Express, 2016, 7, 34-44.

50 M. Scotuzzi, J. Kuipers, D. I. Wensveen, P. de Boer, C. W. Hagen, J. P. Hoogenboom and B. N. G. Giepmans, Sci. Rep., 2017, 7, 45970.

51 R. Carminati, A. Cazé, D. Cao, F. Peragut, V. Krachmalnicoff, R. Pierrat and Y. D. Wilde, Surf. Sci. Rep., 2015, 70, 1-41.

52 C. L. Melcher and J. S. Schweitzer, IEEE Trans. Nucl. Sci., 1990, 37, 161-164.

53 J. Tisler, G. Balasubramanian, B. Naydenov, R. Kolesov, B. Grotz, R. Reuter, J.-P. Boudou, P. A. Curmi, M. Sennour, A. Thorel, M. Börsch, K. Aulenbacher, R. Erdmann, P. R. Hemmer, F. Jelezko and J. Wrachtrup, ACS Nano, 2009, 3, 1959-1965.

54 Y. Lu, J. Zhao, R. Zhang, Y. Liu, D. Liu, E. M. Goldys, X. Yang, P. Xi, A. Sunna, J. Lu, Y. Shi, R. C. Leif, Y. Huo, J. Shen, J. A. Piper, J. P. Robinson and D. Jin, Nat. Photonics, 2014, 8, 32-36.

55 M. W. T. Werten, W. H. Wisselink, T. J. Jansen-van den Bosch, E. C. de Bruin and F. A. de Wolf, Protein Eng., Des. Sel., 2001, 14, 447.

56 C. A. Schneider, W. S. Rasband and K. W. Eliceiri, Nat. Methods, 2012, 9, 671-675.

57 P. Thévenaz, U. Ruttimann and M. Unser, IEEE Trans. Image Process., 1998, 7, 27-41. 